\title{
Elucidation of The Behavioral Program and Neuronal Network Encoded by Dorsal Raphe Serotonergic Neurons
}

\author{
Daniel J Urban ${ }^{1,6}$, Hu Zhu ${ }^{1,6}$, Catherine A Marcinkiewcz ${ }^{2}$, Michael Michaelides ${ }^{3}$, Hidehiro Oshibuchi ${ }^{4}$, \\ Darren Rhea ${ }^{4}$, Dipendra K Aryal', Martilias S Farrell', Emily Lowery-Gionta ${ }^{2}$, Reid HJ Olsen', \\ William C Wetsel ${ }^{5}$, Thomas L Kash ${ }^{2}$, Yasmin L Hurd ${ }^{3}$, Laurence H Tecott ${ }^{*, 4}$ and Bryan L Roth*, I \\ 'Department of Pharmacology and National Institute of Mental Health Psychoactive Drug Screening Program, Chapel Hill, NC, USA; ${ }^{2}$ Bowles \\ Center for Alcohol Studies and Department of Pharmacology, School of Medicine, University of North Carolina, Chapel Hill, NC, USA; ${ }^{3}$ Departments \\ of Psychiatry and Neuroscience, Icahn School of Medicine at Mount Sinai, New York, NY, USA; ${ }^{4}$ Department of Psychiatry, University of California, \\ San Francisco, CA, USA; ${ }^{5}$ Departments of Psychiatry and Behavioral Sciences, Cell Biology, and Neurobiology, Duke University Medical Center, \\ Durham, NC, USA
}

\begin{abstract}
Elucidating how the brain's serotonergic network mediates diverse behavioral actions over both relatively short (minutes-hours) and long period of time (days-weeks) remains a major challenge for neuroscience. Our relative ignorance is largely due to the lack of technologies with robustness, reversibility, and spatio-temporal control. Recently, we have demonstrated that our chemogenetic approach (eg, Designer Receptors Exclusively Activated by Designer Drugs (DREADDs)) provides a reliable and robust tool for controlling genetically defined neural populations. Here we show how short- and long-term activation of dorsal raphe nucleus (DRN) serotonergic neurons induces robust behavioral responses. We found that both short- and long-term activation of DRN serotonergic neurons induce antidepressant-like behavioral responses. However, only short-term activation induces anxiogenic-like behaviors. In parallel, these behavioral phenotypes were associated with a metabolic map of whole brain network activity via a recently developed non-invasive imaging technology DREAMM (DREADD Associated Metabolic Mapping). Our findings reveal a previously unappreciated brain network elicited by selective activation of DRN serotonin neurons and illuminate potential therapeutic and adverse effects of drugs targeting DRN neurons. Neuropsychopharmacology (2016) 4I, |404-14I5; doi:10.1038/npp.2015.293; published online 14 October 20I5
\end{abstract}

\section{INTRODUCTION}

The brain's serotonergic network is a large and complex efferent system that controls a highly diverse range of behavioral and physiological processes, including perception, mood, anxiety, food intake, and circadian rhythm (Berger et al, 2009). Brain serotonergic neurons are distributed in a series of predominantly midline raphe nuclei extending from the midbrain to the caudal medulla and projecting widely throughout the brain and spinal cord (Jacobs and Azmitia, 1992). The midbrain dorsal raphe nucleus (DRN) is the largest of the raphe nuclei, and DRN serotonergic neurons project rostrally to provide the predominant serotonergic innervation of the forebrain (Vertes, 1991; Jacobs and Azmitia, 1992; Waselus et al, 2012). Dysregulation of DRN serotonergic neurotransmission has been implicated in many

* Correspondence: Dr LH Tecott, Department of Psychiatry, University of California, San Francisco, CA 94I43, USA, Tel: 415576 7858, Fax: 415 476 7838, E-mail: Larry.Tecott@ucsf.edu or Dr BL Roth, Department of Pharmacology, UNC Chapel Hill Medical School, NIMH Psychoactive Drug Screening Program, Chapel Hill, NC 27599, USA, Tel: + I 919966 7535, Fax: + 919843 5788, E-mail: bryan_roth@med.unc.edu

${ }^{6}$ These authors contributed equally to this work.

Received 29 June 2015; revised 18 August 2015; accepted 18 August 2015; accepted article preview online 18 September 2015 psychiatric illnesses, such as anxiety and depressive disorders (Graeff et al, 1996; Underwood et al, 1999; Lowry et al, 2008). Additionally, enhancement of DRN serotonin (5-HT) release has been proposed to be responsible for the actions of some antidepressants (Stamford et al, 2000).

Despite the fact that many commonly prescribed and abused serotonergic drugs (eg, fluoxetine, 3,4-methylenedioxy-methamphetamine) globally modulate serotonergic neurotransmission, the precise mechanism(s) responsible for both their therapeutic and adverse effects remain unclear. To address this challenge, we employed an approach enabling selective control of specific serotonergic nuclei. Selective manipulation of the DRN serotonergic neurons has been proven difficult as many (50-75\%) neurons in the DRN are non-serotonergic (Halberstadt and Balaban, 2008). Additionally, although optogenetic approaches are useful for the acute (eg, seconds-minutes) control of specific 5-HT neurons, optogenetic approaches are not particularly suited for mimicking the types of long-term modulation of 5-HT neuronal activity induced by drugs which exert their actions over a prolonged period of time.

Here we show that the hM3Dq DREADD (Designer Receptor Exclusively Activated Designer Drug), which is coupled to Goq signaling pathway and acted as a global neuronal activation tool (Armbruster et al, 2007; Rogan and 
Roth, 2011), remotely controls DRN serotonergic neurons for both short (hours) and long (weeks) duration studies. We discovered that short- and long-term activation of DRN 5-HT neurons induces both similar and divergent behavioral programs, which mimic multiple actions of antidepressant drugs. In parallel, we associated these behavioral changes with alterations in whole brain metabolic activity using a newly developed technology (eg, DREADD Assisted Metabolic Mapping (DREAMM); Michaelides et al, 2013; Michaelides and Hurd, 2015).

\section{MATERIALS AND METHODS}

\section{Animal Subjects and General Procedures}

All procedures were conducted in accordance with the Guide for the Care and Use of Laboratory Animals, as adopted by the National Institutes of Health, and with approval of the Institutional Animal Care and Use Committees at the University of North Carolina (UNC), Duke University, and the University of California at San Francisco. Adult, agematched, male Slc6a4-Cre mice (MMRRC: Stock number017260UCD) were used for all in vivo and ex vivo experiments.

\section{Stereotaxic Injection}

Adult (22-30 g) male Slc6a4-Cre males (017260UCD, MMRRC) were used in current study. Unless described otherwise, all mice were maintained on a standard 12-h light/ dark cycle with ad libitum access to food and water. Mice were anesthetized with a ketamine $(100 \mathrm{mg} / \mathrm{kg})$ and xylazine $(10 \mathrm{mg} / \mathrm{kg})$ solution and placed into a stereotaxtic frame (Kopf Instruments). Mice were bilaterally injected with 0.75-1 $\mu \mathrm{l}$ (depending on viral titer) of adeno-associated virus (AAV) into the dorsal raphe using the following stereotaxtic coordinates: $-4.6 \mathrm{AP}, \pm 1.93 \mathrm{ML}$, and $-3.46 \mathrm{DV}$ at an angle of $30^{\circ}$. Dorsal raphe neurons were transduced with virus encoding the hM3Dq-mCherry or EGFP in reverse orientation flanked by two lox sites (lox P and lox 2722) under the control of the human synapsin (SYN1) promoter. Following surgery, mice were individually housed and monitored for signs of complications until they recovered from surgery. We performed all experiments $4-16$ weeks after surgery. All viral constructs were packaged by the UNC Vector Core Facility. After all experiments, each mouse was perfused and validated for injection efficiency and viral expression.

\section{Immunohistochemistry}

Mice were anesthetized with tribromoethanol and then perfused with $4 \%$ paraformaldehyde (PFA) in PBS. Brains were dissected and postfixed overnight at $4{ }^{\circ} \mathrm{C}$ in $4 \%$ PFA, followed by dehydration in a $30 \%$ sucrose solution in PBS for $48 \mathrm{~h}$. Brain sections $(40 \mu \mathrm{m})$ were permeabilized for $20 \mathrm{~min}$ with $0.3 \%$ Triton X-100 in PBS and then blocked in blocking buffer (PBS containing 0.3\% Triton X-100, 2\% normal goat serum, and $3 \%$ bovine serum albumin) for $1 \mathrm{~h}$ at room temperature. Slides were incubated with first antibody in blocking buffer overnight at $4{ }^{\circ} \mathrm{C}$. Primary antibodies used in the studies were: anti-RFP (mouse, 1:1000, Abcam), anti-5-HT (rabbit, $1: 500$, Immunostar) (for cell bodies) and
$1: 5000$ (Sigma-Aldrich) (for axons), and anti-GFP (mouse, $1: 500$, Abcam). Slides were washed and incubated with secondary fluorescent-conjugated antibodies $(1: 250$, Invitrogen) at room temperature (RT) for $1 \mathrm{~h}$. After an additional three washes, slides were mounted and fluorescent images were collected on either a Nikon 80i Research Upright Microscope (Nikon) equipped with the Surveyor Software with TurboScan (Objective Imaging) or an Olympus Fluoview FV1000 Laser Scanning Confocal Microscope (Olympus). The images of coronal slices were analyzed using the Image-J software (NIH).

\section{Slice Electrophysiology}

Brain slices were prepared for slice electrophysiology as previously described (Marcinkiewcz et al, 2014). Briefly, brains were sectioned at $0.07(\mathrm{~mm} / \mathrm{s})$ to obtain $300 \mu \mathrm{m}$ coronal slices of the DRN. In a recording chamber, slices were submerged in normal, oxygenated aCSF $\left(28-30^{\circ} \mathrm{C}\right.$, $2 \mathrm{ml} / \mathrm{min}$ flow rate) for at least $30 \mathrm{~min}$ before performing whole-cell patch clamp experiments. Borosilicate electrodes with a pipette filled with internal solution ((in $\mathrm{mM}): 135$ KCl-gluconate, $5 \mathrm{NaCl}, 2 \mathrm{MgCl}_{2}, 10 \mathrm{HEPES}, 0.6 \mathrm{EGTA}$, 4 ATP, and 0.4 GTP, $\mathrm{pH}=7.35,290 \mathrm{mOsmol}$ ) were used to patch cells in the DRN. Signals were acquired using a Multiclamp 700B amplifier and analyzed with the Clampfit 10.3 software (Molecular Devices, Sunnyvale, CA, USA). The effects of clozapine-N-oxide (CNO) were determined in current clamp mode. After a 5-min stable baseline, $\mathrm{CNO}$ $(10 \mu \mathrm{M})$ was bath applied for $10 \mathrm{~min}$ while recording changes in membrane potential. For excitability experiments, the current threshold (rheobase) necessary to induce cell firing were determined in current clamp mode using a current ramp protocol from 0 to $100 \mathrm{pA}$. Next, a 10-pA current step protocol from 0 to $200 \mathrm{pA}$ was applied, from which V-I plots were determined (ie, the number of action potentials $v s$ current). Synaptic transmission was assessed in voltage clamp mode by using a cesium methanesulfonate-based intracellular solution. Spontaneous excitatory postsynaptic currents (sEPSCs) were recorded at $-55 \mathrm{mV}$ (the reversal potential for $\mathrm{GABA}_{\mathrm{A}}$-mediated events) and spontaneous postsynaptic inhibitory currents (sIPSCs) were recorded at $+10 \mathrm{mV}$ (the reversal potential for $\mathrm{AMPA}_{\mathrm{R}}$-mediated events). After a 4-min baseline, we averaged the next two, 2-min files to obtain the frequency and amplitude of sEPSCs, sIPSCs, and the ratio of sEPSCs to sIPSCs (E/I ratio). For each cell, we then determined the synaptic drive ratio (EPSC frequency $\times$ amplitude)/(IPSC frequency $\times$ amplitude). The average delta MP for hM3Dq and EGFP cells after CNO was analyzed using a one-sample $t$-test in which the mean was compared with a theoretical mean of zero. Resting membrane potential (RMP), rheobase, and E/I ratios were analyzed using Student's $t$-tests. V-I plots were analyzed using a two-way analysis of variance (ANOVA) with viral vector and current as variables.

\section{Behavioral Studies}

For acute $\mathrm{CNO}$ experiments, each behavioral paradigm was separated at least 1 week for a wash-out period. 
Locomotor activity and center zone time. Locomotor activity was assessed under standardized environmental conditions in $40 \times 40 \times 30 \mathrm{~cm}^{3}$ Plexiglas chambers with a grid of photo-beams spaced at $1.52 \mathrm{~cm}$ (VersaMax system; AccuScan Instruments). Locomotor activity was recorded for $1 \mathrm{~h}$. Data were extracted using the Fusion software (AccuScan Instruments) as described (Farrell et al, 2013).

Light-dark emergence test. Light and dark preference was assessed under standardized environmental conditions in $40 \times 40 \times 30 \mathrm{~cm}^{3}$ Plexiglas chambers as described above, fit with a $16^{\prime \prime} L \times 8^{\prime \prime} H \times 5^{\prime \prime} W$ dark chamber on one side (VersaMax system; AccuScan Instruments). Time spent in the light, dark, and doorway zones were recorded for $10 \mathrm{~min}$. Data were extracted using the VersaMap software (AccuScan Instruments).

Forced swim. Mice were individually placed into a Plexiglas cylinder ( $21 \mathrm{~cm}$ diameter, $28 \mathrm{~cm}$ height) containing $15 \mathrm{~cm}$ water $\left(25 \pm 0.5^{\circ} \mathrm{C}\right)$ and were monitored for $6 \mathrm{~min}$. Mobility, high mobility, and immobility bouts were analyzed using EthoVision XT7 for the last 4 min of the test. After the swim session, mice were dried and placed in their home cage surrounded by a cotton cloth. The water was changed in between each animal.

Novelty-suppressed feeding. Food was removed from the home cage $24 \mathrm{~h}$ before testing. Mice were weighed just before food deprivation and again before testing to assess body weight loss. For testing, mice were placed into a brightly lit open field $(40 \mathrm{~cm} \times 40 \mathrm{~cm} \times 30 \mathrm{~cm}$ white plastic boxes containing $2 \mathrm{~cm}$ of bedding) with a food pellet at the center. Latency to begin eating the food was recorded. The total amount of food consumed during a $5 \mathrm{~min}$ period after returning to the home cage was measured to test whether feeding differences in the novel environment were due to differences in hunger/motivation. These studies were performed after the first $4 \mathrm{~h}$ of the light cycle.

Home cage monitoring (HCM) system. A detailed description of the HCM hardware has been reported elsewhere (Goulding et al, 2008). Briefly, all animals were acclimated to the HCM cages for 1 week, and then baseline data (days 8-15) were collected. After that, CNO was provided in the drinking water at a concentration of $40 \mathrm{mg} / \mathrm{l}$. Chronic CNO treatment data were collected from day 22 to day 29. Data collected during the 7-day periods (days 8-15 and 22-29) were used for within-subject comparisons of behavioral patterns at baseline vs during $\mathrm{CNO}$ treatment, respectively. The impact of DRN serotonergic neuronal activation on exploratory behavior was examined during the $\mathrm{CNO}$ chronic treatment period. We examined the responses of $\mathrm{CNO}-$ treated Slc6a4-hM3Dq and Slc6a4-EGFP mice to a novel object placed within their HCM cages. Four hours prior to the dark cycle onset, a metal binder clip ( 2 inch wide, 1 inch capacity; Officemate International) was affixed to the center of the long cage wall opposite the housing niche for a period of $4 \mathrm{~h}$. Exploratory responses to novel object presentation were assessed by analyzing locomotor paths and determining the amount of time animals spent within a $7 \mathrm{~cm}$ radius of the object during the first hour of exposure.

DREADD-assisted metabolic mapping. Mice were transported to the $\mu \mathrm{PET}$ facility the day prior to each scan to ensure habituation to the transportation procedure and environment. All mice were fasted overnight to attain consistency in blood glucose levels as abnormal blood glucose levels interfere with $\left[{ }^{18} \mathrm{~F}\right]$ fluorodeoxyglucose (FDG) uptake (Wong et al, 2011). The following morning mice received an injection (i.p.) of either vehicle or CNO $(2 \mathrm{mg} / \mathrm{kg})$. This occurred four times, twice before (acute-vehicle and acute-CNO) and twice after 3 weeks of daily $(5 \mathrm{mg} / \mathrm{kg}$, p.o.) $\mathrm{CNO}$ exposure (chronic-vehicle and chronic CNO). Therefore, each mouse was scanned four times. For each acute and chronic phase, vehicle and $\mathrm{CNO}$ scans were separated by two days. For acute phase scans, vehicle preceded $\mathrm{CNO}$ while for chronic phase scans, $\mathrm{CNO}$ preceded the vehicle (for the purpose of minimizing withdrawal effects owing to disruption of daily $\mathrm{CNO}$ administration after chronic exposure). Twenty minutes after vehicle or $\mathrm{CNO}$ injections, mice received an injection (i. p.) of $\sim 0.2 \mathrm{mCi}$ of FDG and were immediately placed in an open-field arena $\left(40.64 \times 40.64 \times 40.64 \mathrm{~cm}^{3}\right)$ coupled to an activity monitoring system (Truscan, Coulbourn Instruments). Thirty minutes later, mice were anesthetized with $1.5 \%$ isoflurane, placed in a prone position on the bed of an R4 microPET rodent scanner (Siemens Medical Solutions), and scanned using a 20-min static acquisition protocol. Scanning procedures, image processing, and analysis were performed as previously described (Michaelides et al, 2010, 2013).

\section{RESULTS}

\section{Selective Expression of hM3Dq DREADD in Serotonergic Neurons in DRN}

To selectively express hM3Dq DREADD in serotonergic neurons in the DRN, we generated a rAAV8 vector construct containing a double-floxed inverted open-reading frame (DIO) sequence encoding hM3Dq-mCherry driven by a human synapsin promoter. The inverted hM3Dq-mCherry was then recombined into a correct orientation only in the cre-recombinase-expressing cells (Sohal et al, 2009). We stereotaxically injected this cre-dependent AAV8 virus (Krashes et al, 2011) into the DRN of Slc6a4-Cre mice, in which cre-recombinase was expressed in adult serotonergic neurons (Gong et al, 2007) (Figure 1a). Three weeks after injection, immunofluorescence staining was performed to determine the co-localization and transduction efficiency of DREADD virus. Immunofluorescence microscopy revealed that hM3Dq DREADD was selectively expressed in DRN (Figure $1 \mathrm{~b})$ and $96.7 \pm 1.6 \%(N=3670$ cells $)$ of hM3Dq expressing neurons co-localized with 5-HT positive neurons with a $64.2 \pm 5.3 \%(N=3670$ cells $)$ transduction efficiency in DRN 5-HT-containing neurons (Figure 1c). A similar degree of co-localization and transduction efficiencies were also observed in Slc6a4-Cre mice stereotaxically injected with a control cre-dependent EGFP AAV virus $(97.7 \pm 1.2 \%$ co-localization, $N=2035$ cells; $73.5 \pm 4.6 \%$ transduction efficiency, $N=2035$ cells; Supplementary Figure S1). In 
a

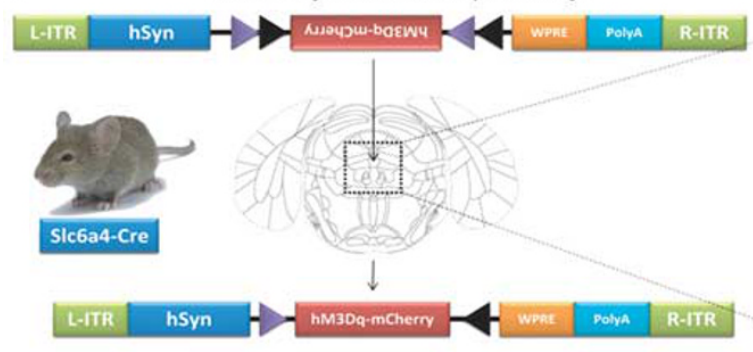

b

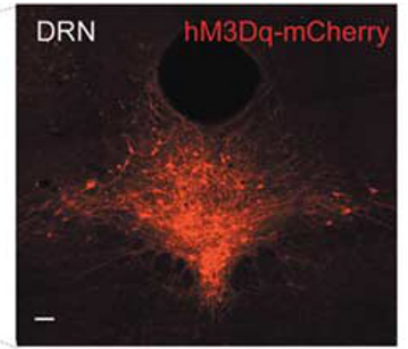

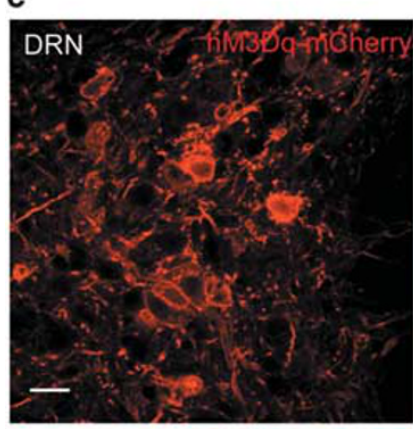
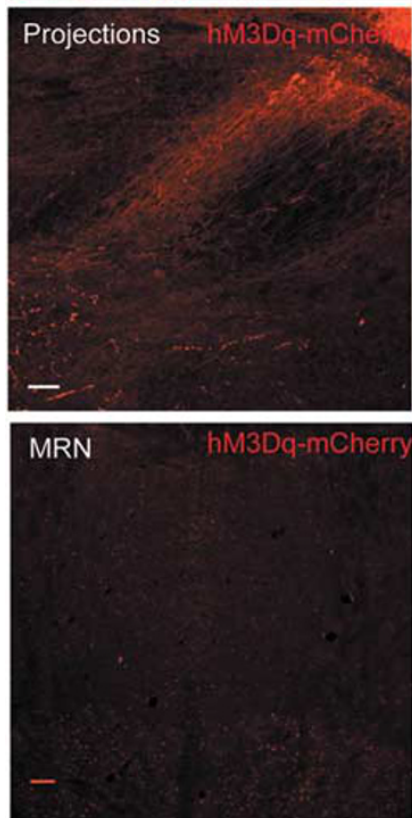
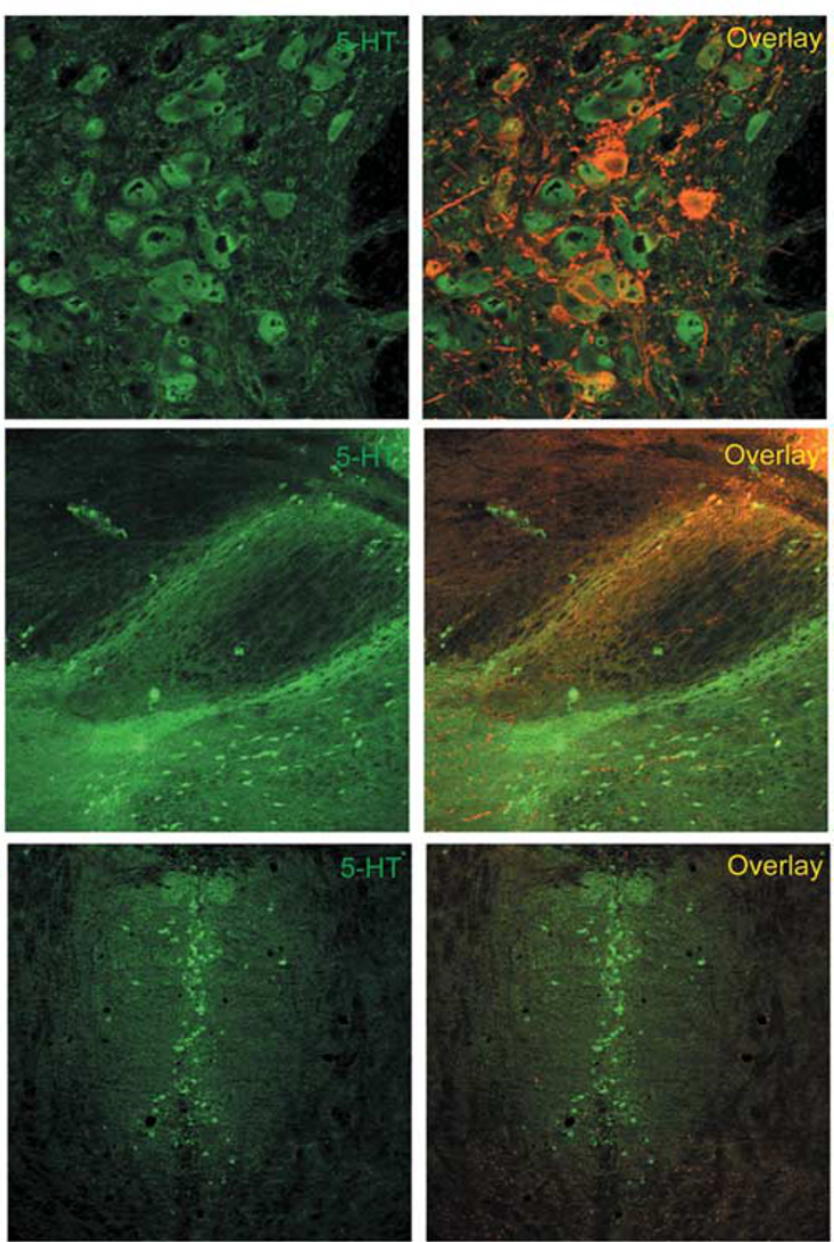

Figure I Selective expression of the hM3Dq DREADD in DRN serotonergic neurons of SIc6a4-Cre mice. (a) Schematic demonstration of the design and stereotaxic injection of the cre-dependent hM3Dq-mCherry AAV driven off the human synapsin promoter. (b) mCherry fluorescence in the DRN after injections of the AAV-hSyn-DIO-hM3Dq-mCherry virus into Slc6a4-Cre mice (scale: $200 \mu \mathrm{m}$ ). (c) Upper panels: co-localization of mCherry (Red) and 5-HT (Green) in the dorsal raphe nucleus (DRN). The hM3Dq DREADD receptor is expressed in the plasma membrane while 5-HT is in the cytoplasm (scale: $10 \mu \mathrm{m}$ ). Middle panels: co-localization of hM3Dq receptor (Red) with 5-HT-positive axons (Green) projecting from the DRN (scale: $50 \mu \mathrm{m}$ ). Lower panels: lack of expression and co-localization of hM3Dq receptor (Red) with 5-HT-positive neurons (Green) in the median raphe nucleus (MRN) (scale: $50 \mu \mathrm{m}$ ).

addition, 5-HT positive axons emanating from the DRN co-localized with $\mathrm{hM} 3 \mathrm{Dq}$ receptors, thus demonstrating efficient trafficking of the hM3Dq receptor to the axons of DRN serotonergic neurons (Figure 1c). Importantly, there was negligible expression of $\mathrm{hM} 3 \mathrm{Dq}$ in 5-HT-positive neurons of the median raphe nucleus (hM3Dq: $2.3 \pm 3.0 \%$; $N=586$ cells; Figure 1c), further highlighting selective expression of hM3Dq in 5-HT-positive neurons in DRN.
Acute Activation of DRN Serotonergic Neurons Induces Anxiogenic and Antidepressant Drug-Like Behavioral Responses

After verifying selective expression of DREADD receptors in DRN serotonergic neurons, we assessed the electrophysiological properties of these neurons by activating hM3Dq receptors (Figure 2a). After $\mathrm{CNO}$ exposure, a significant increase in membrane potential was observed in 


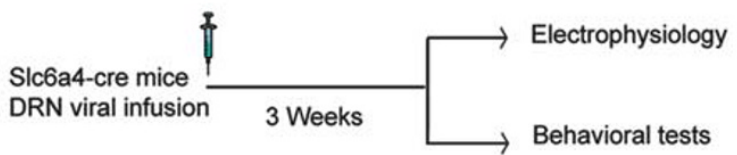

b

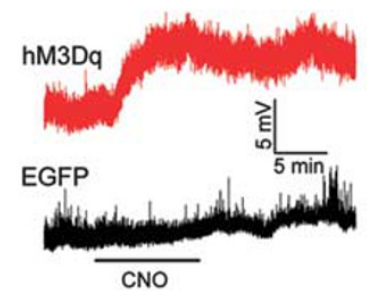

e

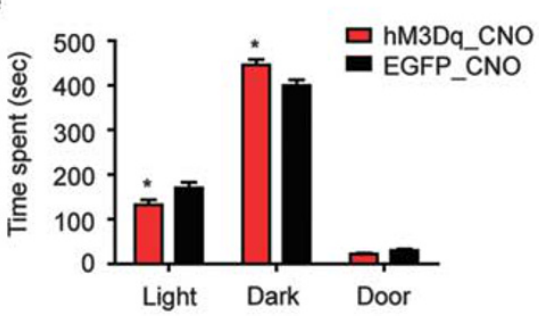

h

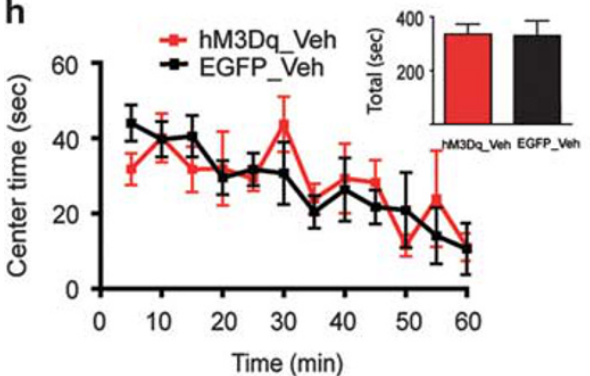

C

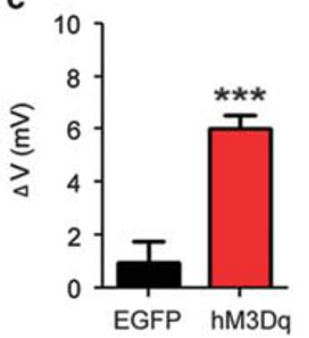

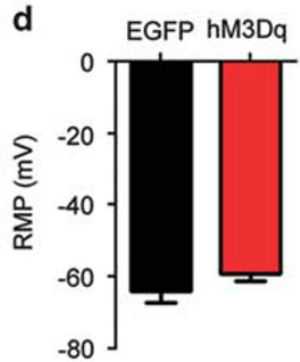

f
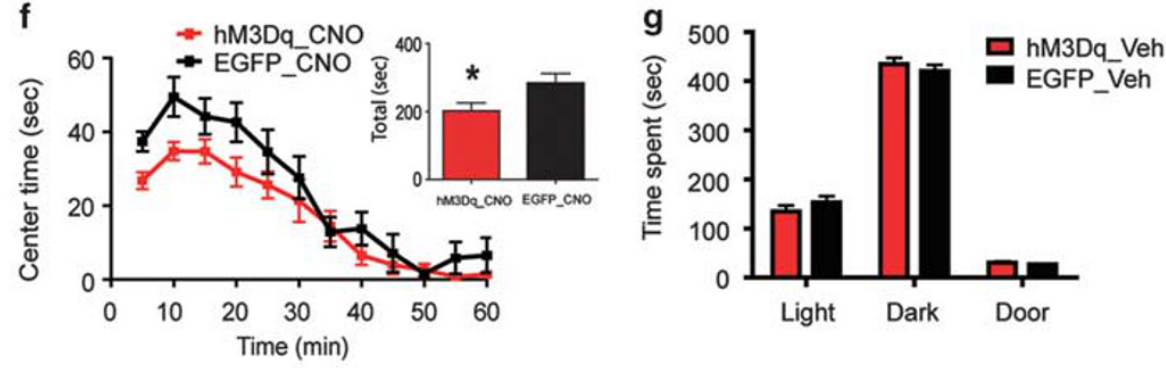

i

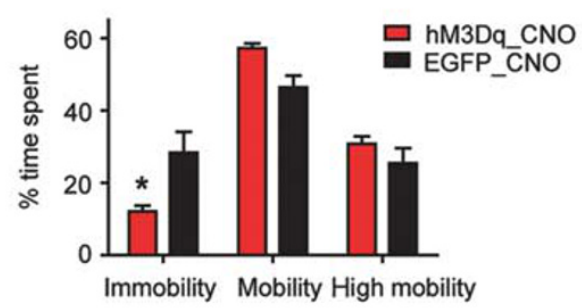

j

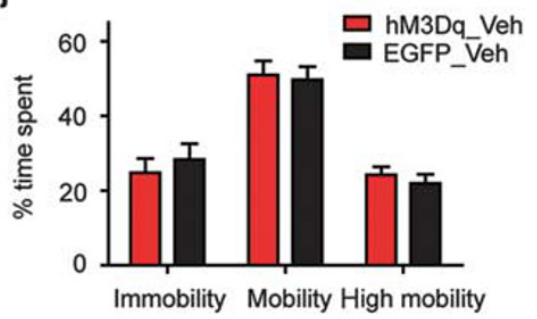

Figure 2 Acute activation of DRN serotonergic neurons induces anxiogenic and antidepressant drug-like behavioral responses. (a) Schematic diagram of acute CNO treatment experiments. (b) Representative trace and (c) bar graph showing average change in membrane potential in hM3Dq ( $\mathrm{h}=5$ ) and EGFP $(N=5)$ neurons. After a 5-min baseline, CNO $(10 \mu \mathrm{M})$ was bath applied for $10 \mathrm{~min}$. **** $p<0.00$ I (d) Average resting membrane potential (RMP) of hM3Dq and EGFP neurons. (e) Time spent in the lighted, darkened, or doorway compartments by Slc6a4-hM3Dq $(N=15)$ and Slc6a4-EGFP (N=I3) mice over a I0-min period following acute administration of CNO (*p<0.05). (f) Time spent in the center of an open field by Slc6a4-hM3Dq (N= I6) and SIc6a4-EGFP $(\mathrm{N}=16)$ mice shown in 5-min binned intervals after acute $\mathrm{CNO}$ administration. Inset: Total center time for the 60-min trial $(* p<0.05)$. (g) Time spent in the lighted, darkened, or doorway compartments by SIc6a4-hM3Dq $(N=8)$ and SIc6a4-EGFP $(N=8)$ mice over a I0-min period following acute administration of vehicle. (h) Time spent in the center of an open field by Slc6a4-hM3Dq $(N=8)$ and Slc6a4-EGFP $(N=8)$ mice in 5 -min binned intervals after acute vehicle administration. Inset: Cumulative center time for the 60-min test. (i) Percentage of time Slc6a4-hM3Dq $(N=9)$ and Slc6a4-EGFP (N=9) mice exhibited immobility, mobility, and high mobility during the last 4-min of the forced swim test after acute injection of CNO (* $p<0.05$ ). (j) Percentage of time Slc6a4hM3Dq $(N=9)$ and Slc6a4-EGFP $(N=9)$ mice exhibited immobility, mobility, and high mobility during the last 4-min of the forced swim test after acute administration of vehicle.

hM3Dq-positive DRN neurons $(t(4)=12.06, p=0.0003$, Figure $2 \mathrm{~b}$ and $\mathrm{c}$ ) but not in EGFP-expressing neurons $(t(4)=1.182, p=0.3028)$ - in agreement with many previous studies (Alexander et al, 2009; Krashes et al, 2011, 2014; Garfield et al, 2015). Importantly, hM3Dq-expressing neurons had similar RMPs compared with EGFP-expressing control neurons (Figure 2d).

DRN serotonergic neurons have long been implicated in the actions of both anxiolytics and antidepressants, and we predicted that activation of these neurons would mimic the actions of these drugs. Initially, two anxiolytic paradigms, light-dark emergence test and open-field test, were tested $45 \mathrm{~min}$ after acute CNO (2 mg/kg) administration. As shown in Figure $2 \mathrm{e}$ and $\mathrm{f}$, acute activation of DRN neurons induced relatively modest, though statistically significant, anxiogeniclike responses in light-dark emergence test. CNO-treated Slc6a4-hM3Dq mice displayed a significant reduction of time spent in the lighted chamber and a reciprocal increase of time spent in the darkened chamber (genotype $\times$ chamber, $\mathrm{F}(2,78)=9.16, p=0.0003$, two-way ANOVA Figure 2e). A similar anxiogenic-like response was also manifested by a significantly decreased center time in the open field in 


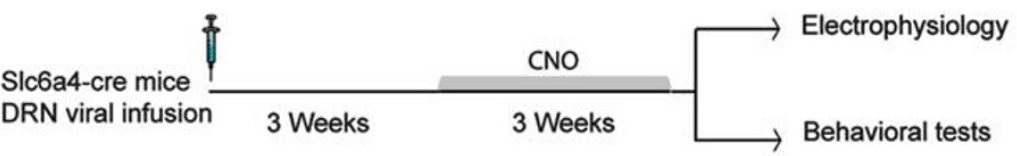

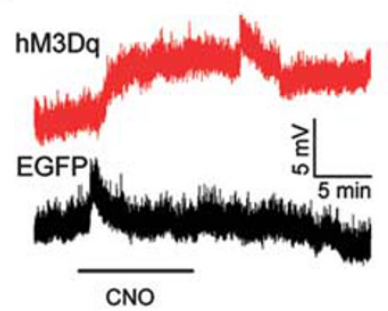

C

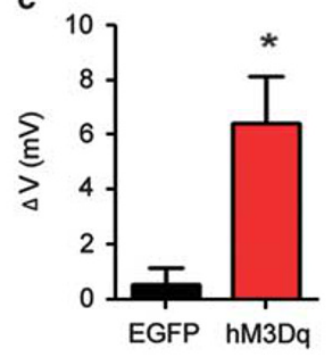

d

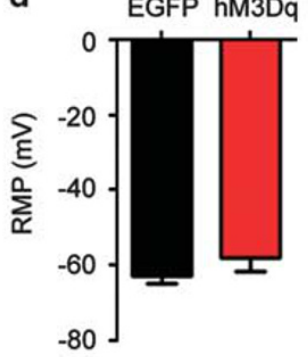

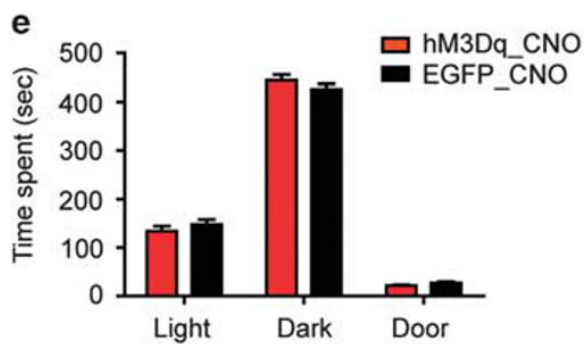
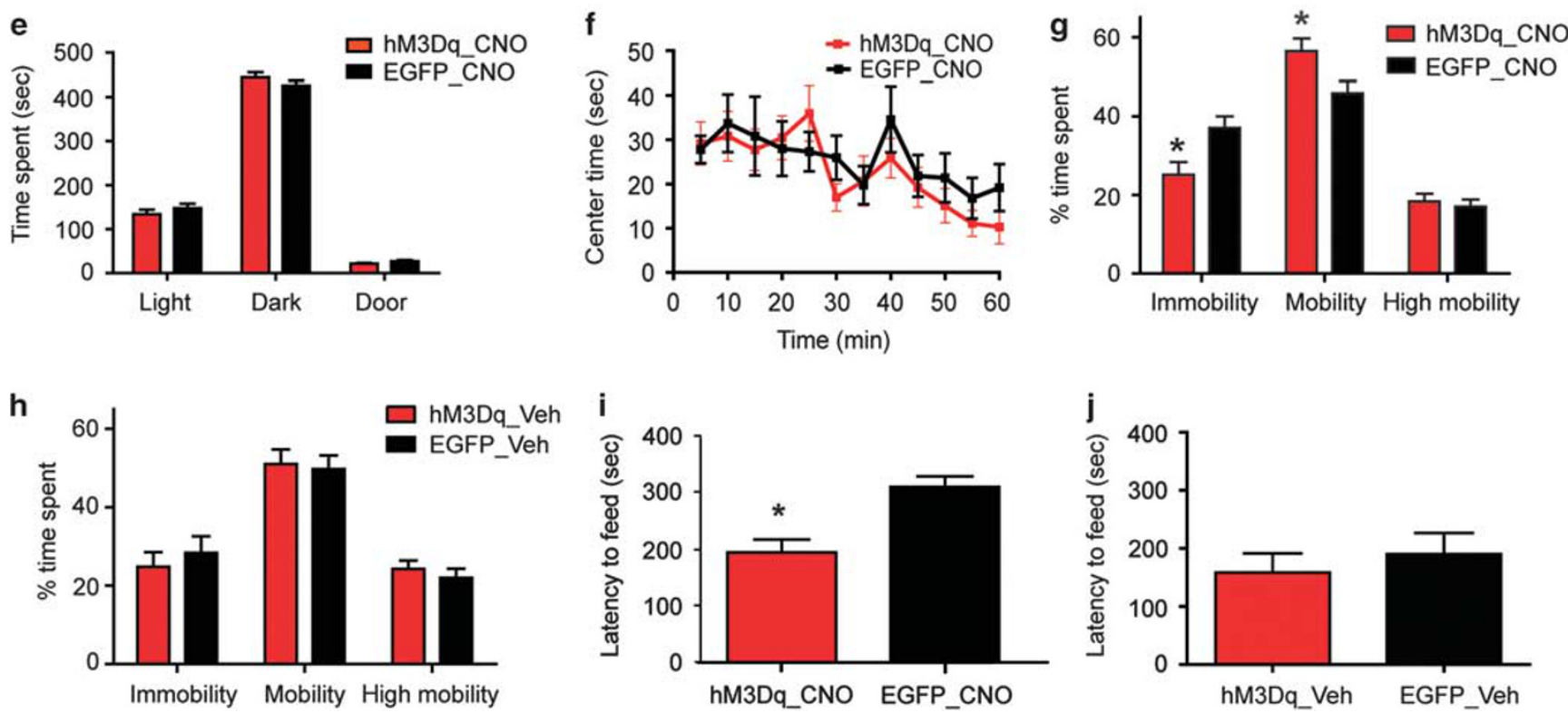

Figure 3 Chronic activation of the DRN serotonergic neurons induces antidepressant-like responses. (a) Schematic diagram of chronic CNO treatment experiments. Animals were chronically administered with CNO $(5 \mathrm{mg} / \mathrm{kg} /$ day $)$ in the drinking water for 3-4 weeks and then tested in the electrophysiology or behavioral paradigms (b) Representative trace and (c) bar graph showing average change in membrane potential in hM3Dq ( $N=4$ ) and EGFP $(\mathrm{N}=8)$ neurons from mice treated chronically with $\mathrm{CNO}(5 \mathrm{mg} / \mathrm{kg} /$ day $)$ for 3 weeks. After a 5 -min baseline, CNO (IO $\mu \mathrm{M})$ was bath applied for IO min $(* p<0.05)$. (d) Average resting membrane potential (RMP) of $\mathrm{hM} \mathrm{Dq}$ and EGFP neurons. (e) Time spent in the lighted, darkened, or doorway compartments by Slc6a4-hM3Dq ( $=17)$ and Slc6a4-EGFP $(N=17)$ mice over a I0-min period. ( $f$ ) Time spent in the center zone of an open field by Slc6a4hM3Dq $(N=17)$ and Slc6a4-EGFP $(N=17)$ mice in 5-min binned intervals. (g) Percentage of time Slc6a4-hM3Dq $(N=13)$ and Slc6a4-EGFP (N= I3) mice engaged in immobility, mobility, and high mobility during the last 4-min of the forced swim test $(* p<0.05)$. (h) Percentage of time Slc6a4-hM3Dq ( $N=9)$ and Slc6a4-EGFP $(N=9)$ mice exhibited immobility, mobility, and high mobility during the last 4-min of the forced swim test in vehicle treatment. (i) Latency to feed by Slc6a4-hM3Dq $(N=17)$ and Slc6a4-EGFP $(N=17)$ mice after administration of CNO in an aversive environment following food deprivation $(* p<0.05)$. (j) Latency to feed by Slc6a4-hM3Dq $(N=8)$ and Slc6a4-EGFP $(N=8)$ mice after administration of vehicle in an aversive environment following food deprivation.

CNO-treated Slc6a4-hM3Dq mice $(t(1,30)=2.246, p=0.0322$, Figure 2f) without alterations of locomotion profiles (Supplementary Figure S2). Importantly, these anxiogenic responses were not observed in vehicle-treated Slc6a4-hM3Dq mice, suggesting that these behaviors were specifically induced by activating hM3Dq DREADD (Figure $2 \mathrm{~g}$ and $\mathrm{h}$ ).

Next, we wondered whether acutely activating the hM3Dq receptors in the DRN could induce antidepressant-like responses in the forced swim test similar to the actions of many clinically effective antidepressants. Following acute $\mathrm{CNO}$ administration, Slc6a4-hM3Dq mice displayed a significant reduction in immobility when compared with Slc6a4-EGFP control mice $(\mathrm{F}(2,48)=5.86, p=0.0006$, Figure 2i) without altering locomotion (Supplementary Figure S2). Again, these antidepressant-like responses were not observed in Slc6a4-hM3Dq mice if vehicle was administered (Figure 2j).

\section{Chronic Activation of DRN Serotonergic Neurons Induces Antidepressant Drug-Like Behavioral Responses}

The serotonergic system is a major target of many antidepressant drugs and in humans it typically requires weeks for optimal effects to be observed (Quitkin et al, 
1984; Lam, 2012). Thus, to better mimic the long-term effects of antidepressants, we decided to take advantage of our chemogenetic system to chronically and non-invasively modulate neuronal activity over a long period of time and then investigate the effects of chronically activating DRN serotonergic neurons on the behaviors of animals. As previously described (Jain et al, 2013), CNO (5 mg/kg/day) was administered continuously via drinking water for 3 weeks (Figure 3a). Then the electrophysiological properties of DRN serotonergic neurons were assessed to determine whether these neurons can still be activated by $\mathrm{CNO}$ after long-term CNO stimulation. Bath application of CNO significantly depolarized $(t(3)=3.842, \quad p=0.0311) \quad h M 3 D q$-expressing neurons but had no effect on EGFP-expressing neurons $(t(7)=0.989, p=0.356$; Figure $3 \mathrm{~b}$ and $c)$. Chronic CNO treatment had no effect on RMP $(t(10)=0.426, p=0.287$, Figure 3d).

After confirming that hM3Dq DREADD can robustly and continuously activate serotonergic neurons, we examined whether chronic activation of DRN serotonergic neurons could induce anxiogenic and antidepressant drug-like responses. We found that chronic $\mathrm{CNO}$ administration had no effect on a variety of anxiety measurements, including the light-dark emergence test (Figure 3e) and center time in the open-field test (Figure 3f). Importantly, chronic $\mathrm{CNO}$ administration had no effect on locomotor activity, center

a Home cage monitoring system (chronic CNO treatment)

Slc6a4-cre mice
DRN viral infusion
Active state behavior
Exploratory behavior

b

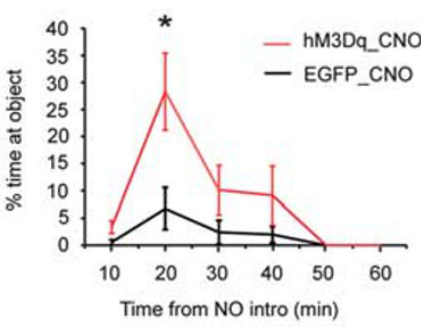

e

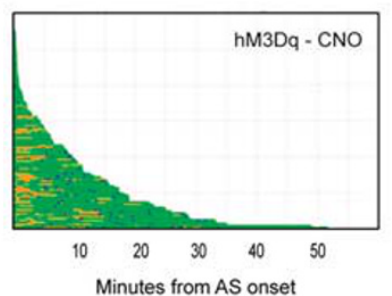

c
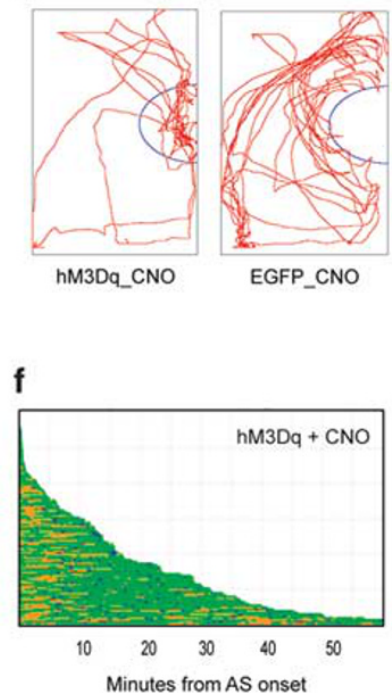

d

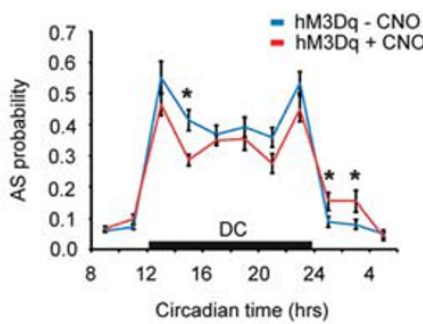

g

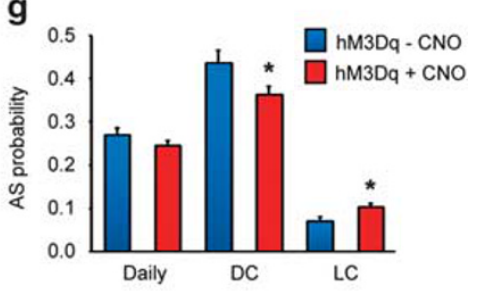

Figure 4 Chronic activation of the DRN serotonergic neurons induces a shift of circadian patterns of behavior. (a) Schematic diagram of experimental procedure of home monitoring system. (b) Percentage of time spent by Slc6a4-hM3Dq $(N=8)$ and Slc6a4-EGFP $(N=8)$ mice in the vicinity of novel object during CNO treatment (*p<0.05). (c) Representative locomotor paths occurring 10-20 min after novel object placement for Slc6a4-hM3Dq and Slc6a4EGFP mice; the vicinity of the novel object indicated by blue hemicircle $(7 \mathrm{~cm}$ radius). (d) Mean active state probability in 2-h time bins for Slc6a4-hM3Dq mice $(\mathrm{N}=8)$ before and during $\mathrm{CNO}$ treatment. Dark cycle $(\mathrm{DC})$ indicated by black bar $(* p<0.05)$. Active states occurring during the first $4 \mathrm{~h}$ of the light cycle for Slc6a4-hM3Dq (N=8) mice, aligned by onset time prior to $(\mathrm{e})$ and during $(f) \mathrm{CNO}$ treatment. Each row indicates an individual's active state in order (top to bottom) of increasing duration. Locomotor, feeding, and drinking behavior indicated in green, orange, and blue, respectively. (g) Mean daily, DC and light cycle (LC) active state probability for Slc6a4-hM3Dq mice $(N=8)$ before and during CNO treatment $(* p<0.05)$.

Figure 5 Metabolic map of whole brain network activity after activation of DRN serotonergtic neurons. (a) DREAMM reveals alterations in anxiety-related regions after acute activation of DRN serotonergic neurons. DREAMM responses after acute injection of CNO combined with an open-field exposure ( $p=0.05$, relative increase (red) and decrease (blue) in FDG uptake; $N=8$, comparison of vehicle vs CNO scans: within-subject comparison). (b) DREAMM reveals decreases in antidepressant-related regions after chronic activation of DRN serotonergic neurons. DREAMM responses after chronic treatment of CNO combined with an open-field test ( $p=0.00$ I, relative decrease (blue) in FDG uptake; $N=8$, comparison of vehicle vs chronic CNO scans: withinsubject comparison). Abbreviations: AOM-anterior olfactory nucleus (medial part), SC - primary somatosensory cortex, MC - primary motor cortex, Pir-piriform cortex, Cg2 - cingulate cortex 2, CT_central thalamic nucleus, MT-medial thalamic nucleus, PAG-periaqueductal gray, IC-inferior colliculus, PV—paraventricular nucleus of the thalamus, $\mathrm{Hb}$ - habenula, TH—-thalamus, DG — dentate gyrus, AC — auditory cortex, DS - dorsal subiculum, LDTg — lateral dorsal tegmental nucleus, DTg_-dorsal tegmental nucleus, MPB — medial parabrachial nucleus, MVe - medial vestibular nucleus, CBN_cerebellar nuclei, and CAI and CA2-areas of hippocampus. 
a PET imaging (acute CNO treatment)

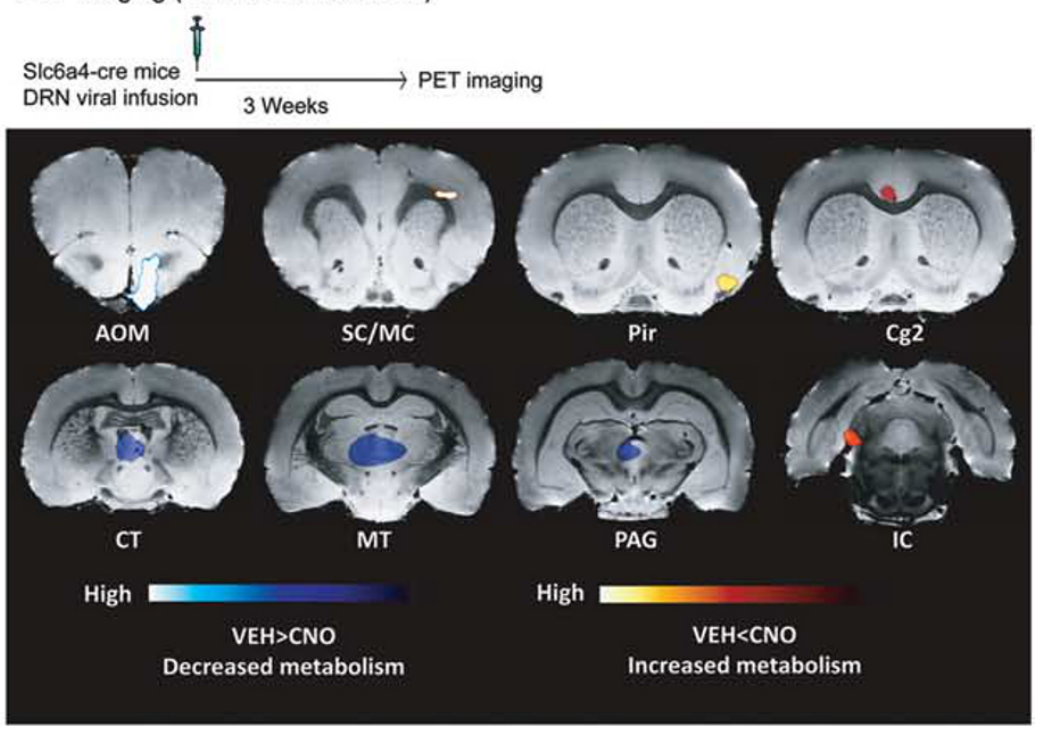

b PET imaging (chronic CNO treatment)

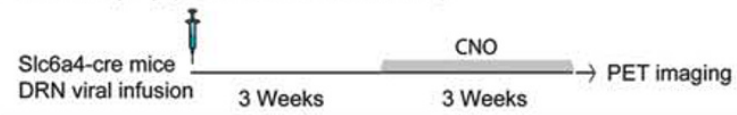

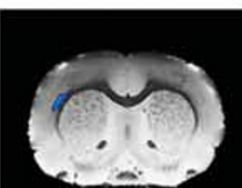

SC

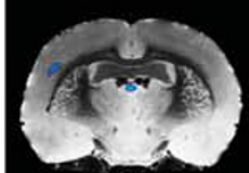

SC PV

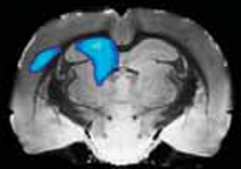

AC SC CA1 DG Hb TH

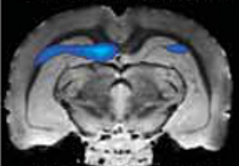

AC CA1 CA2 DG DS

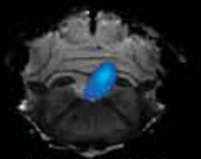

LDTg DTg MPB

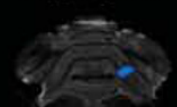

CBN

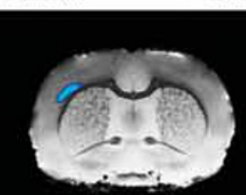

SC

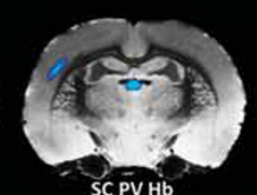

SC PV Hb

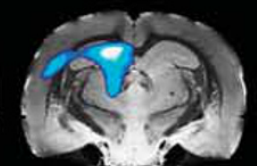

AC SC CA1 CA2 DG HO TH

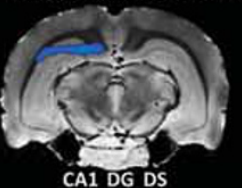

CA1 DG DS

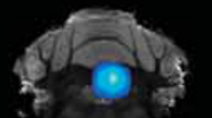

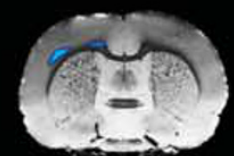

SC MC

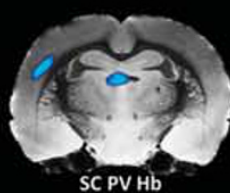

SC PV Hb

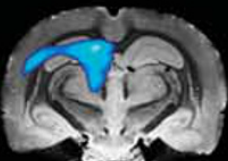

AC CA1 CA2 DG Hb TH

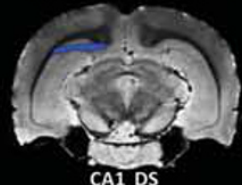

CA1 DS

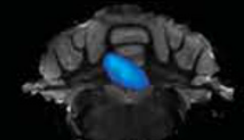

MVe CBN

CBN

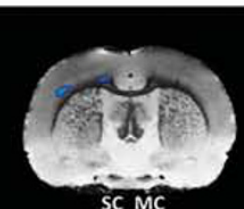

SC MC

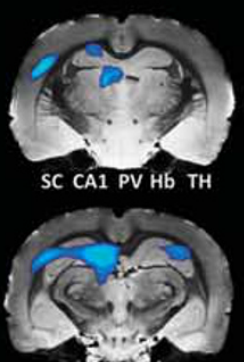

AC CAICAR DG TH
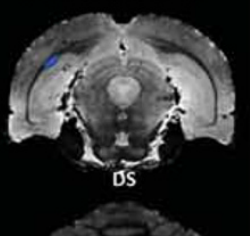

(2)

6. 
time, or light-dark emergence responses in Slc6a4-EGPF control mice (Supplementary Figures S3-S5).

We next examined whether chronic administration of CNO leads to antidepressant-like behavioral responses. As shown in the forced swim test (Figure 3g), a significant decrease in immobility and an increase in mobility were observed in Slc6a4-hM3Dq mice when compared with the Slc6a4-EGFP control mice (genotype $\times$ condition interaction, $\mathrm{F}(2,72)=8.35, p=0.0005)$, demonstrating a robust and prolonged antidepressant-like effect from chronic CNO treatment. Importantly, this antidepressant-like effect was not observed if Slc6a4-hM3Dq mice (another cohort) were in a group in which vehicle-only drinking water was consumed (Figure 3h). There was also no significant difference between chronic CNO and vehicle administration in Slc6a4-EGFP control mice (Supplementary Figure S6). Further characterization of this antidepressant-like phenotype resulting from chronic treatment was evaluated using the noveltysuppressed feeding paradigm (Santarelli et al, 2003; David et al, 2009). Chronic administration of CNO induced a significant reduction in the latency to feed in Slc6a4-hM3Dq mice $(t(32)=3.913, p=0.0004)$ (Figure 3i). However, chronic administration of vehicle failed to induce the reduction in the latency to feed in these mice (another cohort) (Figure $3 \mathrm{j}$ ). There was also no significant difference between chronic CNO and vehicle administration in Slc6a4EGFP control mice (Supplementary Figure S7). Taken together, these data provide further evidence that antidepressant-like behaviors can be induced by chronically activating DRN 5-HT neurons via hM3Dq DREADD.

We next evaluated the effects of chronic CNO administration on the exploratory behaviors of Slc6a4-hM3Dq and Slc6a4-EGFP mice by introducing a novel object into the home cage (Figure 4a). The time spent by $\mathrm{CNO}$-treated Slc6a4-hM3Dq and Slc6a4-EGFP mice within a $7 \mathrm{~cm}$ radius of the object (occupancy time) was then analyzed over $1 \mathrm{~h}$. Time spent in the vicinity of the object was significantly enhanced in Slc6a4-hM3Dq mice, relative to Slc6a4-EGFP mice at the $20 \mathrm{~min}$ time point (genotype $\times$ time $(\mathrm{F}(3,42)=$ 6.39, $p=0.0011$ ), Tukey-Kramer post-hoc test revealed a significant effect $(p=0.032$ at the 20 -min time point; Figure $4 \mathrm{~b}$ ), and the patterns of exploratory interactions were also different between the two groups (Figure 4c). Importantly, there was no significant difference between the two groups in locomotor activities (Supplementary Figure S8).

\section{Chronic Activation of DRN Serotonergic Neurons Alters Circadian Patterns of Activity}

As serotonin has long been implicated in the regulation of sleep and circadian patterns of activity (Monti, 2008), we hypothesized that such effects would be observed during chronic activation of DRN serotonergic neurons. We utilized a quantitative, robust, and automated procedure to assess whether the activation of DRN serotonergic neurons could alter the circadian regulation of physical activity. We monitored two fundamental and mutually exclusive states of animals in a home cage environment: active states (ASs) and inactive states (ISs). In ASs, animals engage in bouts of feeding, drinking, locomotion, and other behaviors. In ISs, animals engage in prolonged episodes of low physical activity (including sleep and resting) occurring exclusively in the nest
(Goulding et al, 2008). We then analyzed these active state behaviors and calculated the active state probability (ASP) in both the dark cycle (DC) and light cycle (LC), a measure which is highly sensitive to circadian rhythm.

For this study, we continuously monitored behavior prior to (days 8-15) and during $\mathrm{CNO}$ treatment (days 22-29) (Figure 4a). We found that chronic CNO treatment shifted activity patterns in Slc6a4-hM3Dq mice-a significant overall reduction of ASP in DC and a significant enhancement of ASP in LC $(\mathrm{CNO} \times$ circadian phase interaction, $\mathrm{F}$ $(10,147)=2.65, p=0.005$, Tukey-Kramer post-hoc testing revealed significant differences $(p<0.023)$ in circadian time at the 15,1 , and $3 \mathrm{~h}$ time points (Figure $4 \mathrm{~d}-\mathrm{g}$ )). Importantly, this circadian shift was not observed in Slc6a4-EGFP control mice with chronic CNO treatment (Supplementary Figure S9). Together, these data indicate that chronic activation of DRN serotonergic neurons alters the circadian regulation of activity in animals.

\section{DREAMM Reveals Discrete Patterns of Brain Metabolic Activity Following Activation of DRN 5-HT Neurons}

In an effort to map profiles associated with acute and chronic DRN serotonin neuronal activation in the open field with regional brain responses, we used DREADD-assisted metabolic mapping (DREAMM), which enables whole-brain circuit dissection in response to cell-type-specific signaling manipulations in behaving rodents (Michaelides et al, 2013). Concurrent with a reduction in time spent in the center of the open field, DREAMM revealed that acute hM3Dq activation of DRN 5-HT neurons led to increased metabolic activity (ie, FDG uptake) in the cingulate cortex (Cg2), the right inferior colliculus (IC), the right piriform (Pir), and primary somatosensory (SC), and motor cortices (MC) (Figure 5a). In contrast, decreases in FDG uptake were observed in the periaqueductal gray, anterior olfactory, and medial and central thalamic areas (MT and CT, respectively) (Figure 5a). CNO was previously shown to be devoid of non-specific effects on FDG uptake (Michaelides et al, 2013). The specificity of DREAMM responses to the behaviors observed in the open field after acute hM3Dq activation were validated using both within-subject (scanned after vehicleadministered open-field sessions) and between-subject controls (Slc6a4-EGFP mice), which did not show changes in time spent in the center of the open field in response to acute DRN serotonin neuron activation.

In contrast with the data from acute 5-HT DRN neuronal activation, DREAMM revealed that chronic DRN 5-HT neuronal activation produced widespread decreases in metabolic activity in many forebrain areas. Interestingly, a large reduction in FDG uptake was observed in hippocampal regions, specifically in CA1, the left dentate gyrus (DG), and the left dorsal subiculum (DS) (Figure 5b). Decreases in FDG uptake were also observed throughout most of the left habenula $(\mathrm{Hb})$ along with areas of the left MT and CT regions (Figure 5b). In addition, FDG uptake was reduced in cortical areas, including the left SC, left primary MC, and left auditory cortex as well as hindbrain regions, such as the medial parabrachial nucleus, dorsal and lateral dorsal tegmental, the medial vestibular, and cerebellar nuclei (Figure 5b). These widespread decreases in DREAMM responses were not observed in Slc6a4-EGFP control mice, 
which revealed no overlapping metabolic changes with CNO-exposed Slc6a4-hM3Dq mice.

To further investigate whether the chronic activation of hM3Dq led to desensitization of hM3Dq during long-term treatment, we applied DREAMM technology following an acute administration of CNO in chronically treated Slc6a4hM3Dq mice. DREAMM revealed an intense increase in FDG uptake in the DRN (Supplementary Figure S10)—supporting our prior observations (Alexander et al, 2009; Krashes et al, 2011) and that of others (Jain et al, 2013; Carter et al, 2014) that $\mathrm{hM} 3 \mathrm{Dq}$ displays robust and continuous activity even with long-term stimulation by $\mathrm{CNO}$-also confirming our ex vivo slice results.

\section{DISCUSSION}

Here we reported that both acute and chronic activation of DRN serotonergic neurons produces a widespread alteration of functional metabolic activity in the mouse brain, which can be associated with certain behavioral phenotypes. Our results provide new insights into the serotonergic regulation of neural pathways relevant to psychoactive drug action and describe a pattern of behavior and brain metabolic activation elicited by selective DRN serotonergic activation.

Both acute and long-term modulation of serotonergic neuronal activity has been implicated in many therapies that alleviate depression and other disorders (Kroenke et al, 2001). In addition, SSRIs have also been demonstrated effective in various animal models, which are utilized as preclinical screens for these drugs (Willner, 1990). Selectively increasing DRN serotonergic neuronal activity revealed analogous antidepressant-like effects in several of these preclinical behavioral tests. In our study, we found that both acute and chronic stimulation of DRN serotonergic neurons produced antidepressant-like behaviors. Interestingly, anxiety-like behaviors were only seen in acute but not with chronic stimulation of DRN serotonergic neurons, which suggests that neuroadaptive changes may occur during chronic stimulation of the serotonergic system (Faure et al, 2006). The neuroadaptive changes in chronic stimulation were further supported by our PET imaging data that was associated with the behavioral phenotypes. Collectively, these data implicate that DRN serotonergic neurons have a critical role in mediating antidepressant-like activities.

Serotonin and circadian systems are reciprocally connected both anatomically and functionally. The suprachiasmatic nucleus (SCN), the primary circadian pacemaker, sends indirect projections to and receives serotonergic innervations from raphe nucleus (Monti, 2008). Serotonergic key signaling molecules SERT, 5-HT1B, 5-HT7, and 5-HT2C receptors are also expressed in the SCN nucleus (Ciarleglio et al, 2011). Increased serotonergic tone is associated with wakefulness but not sleep. Pharmacological and genetic dysfunction of the 5-HT gene network has shown significant influences on circadian behaviors (Ciarleglio et al, 2011). Our study provides direct evidence that chronic stimulation of DRN serotonergic neurons induces alterations in the circadian regulation of behavior, a finding that is notable in light of known perturbations of circadian patterns of behavior in depressive disorders.

Mapping functional brain anatomy associated with acute and chronic hyper-serotonergic states in mice during behavior revealed alterations in regions that are implicated in anxiety and depression. Acute activation during the expression of an anxiogenic phenotype in the open field highlighted a mix of changes in FDG uptake in anxietyrelated and sensory-processing nuclei. In particular, significant increases in functional metabolic uptake of FDG into the left cingulate and right piriform cortices was observed. Importantly, increased cerebral blood flow and glucose uptake in the cingulate cortex region has been strongly linked to anticipatory anxiety in humans (Chua et al, 1999; Javanmard et al, 1999; Straube et al, 2009) and anxiogenic contextual conditioning in rats (Luyten et al, 2012) while increases in FDG uptake in the Pir appear after acute immobilization stress in rats (Sung et al, 2009).

In contrast, chronic activation of DRN 5-HT neurons led to widespread decreases in FDG uptake, specifically in the left dorsal and ventral hippocampal regions (CA1, DG, and DS), $\mathrm{CA} 1$ region of the right hippocampus, left $\mathrm{Hb}, \mathrm{MT}$, and $\mathrm{CT}$ regions as well as areas of the brain stem and cerebellum. Importantly, decreases in FDG metabolism in hippocampal regions have been observed in patients who respond to SSRI treatment (Mayberg et al, 2000; Kennedy et al, 2001; Seminowicz et al, 2004). In addition to the hippocampus, decreased metabolism in the $\mathrm{Hb}$ has been linked to antidepressant drug actions (Shumake et al, 2010). Indeed, the $\mathrm{Hb}$ has been successfully targeted by deep brain stimulation (DBS) to suppress activity in a patient who failed to respond to antidepressant therapies (Sartorius et al, 2010), and there is currently an ongoing clinical trial using lateral Hb DBS for treatment-resistant depression (NCT01798407). Our findings demonstrate the pervasive consequences of selectively activating a relatively small group of serotonergic neurons in the dorsal raphe, further elucidating how 5-HT can induce both anxiety-like activity as well as antidepressant-like responses by differentially modulating the same neurons.

In conclusion, activation of DRN serotonergic neurons is sufficient to reproduce the effects of antidepressants that target 5-HT systems globally. Additionally, we metabolically mapped the effects of selective DRN serotonergic activation potentially delineating regional circuitry implicated in depression. Beyond the insights into the serotonergic regulation of behavior provided by this work, our findings highlight the power of DREADD-based chemogenetic strategies for dissecting the functional consequences of activation of complex neural pathways.

\section{FUNDING AND DISCLOSURE}

This work was supported by the National Institute of Mental Health (R01MH61887, U19MH82441, and U01MH105892, R21 MH079145), National Institute of Alcohol Abuse and Alcoholism (Grants AA11034, AA07574, AA07611), National Institue on Drug Abuse (NIDA, DA030359, DA015446) and the National Institute of Child Health and Human Development (Grant no. P30 HD03110). MM was supported by the NIDA Postdoctoral Training Program at Mount Sinai (DA007135). BLR has consulted for Otsuka Pharmaceuticals, Merck, Sunovion, Albany Molecular Research, Pfizer Pharmaceuticals, Finnegan, Henderson, Farabow, Garrett and Dunner, and Venrock. BLR receives compensation for serving as Associate Editor of the Journal of Clinical Investigation. The authors declare no conflict of interest. 


\section{REFERENCES}

Alexander GM, Rogan SC, Abbas AI, Armbruster BN, Pei Y, Allen JA et al (2009). Remote control of neuronal activity in transgenic mice expressing evolved $\mathrm{G}$ protein-coupled receptors. Neuron 63: 27-39.

Armbruster BN, Li X, Pausch MH, Herlitze S, Roth BL (2007). Evolving the lock to fit the key to create a family of G proteincoupled receptors potently activated by an inert ligand. Proc Natl Acad Sci USA 104: 5163-5168.

Berger M, Gray JA, Roth BL (2009). The expanded biology of serotonin. Annu Rev Med 60: 355-366.

Carter ME, Soden ME, Zweifel LS, Palmiter RD (2014). Genetic identification of a neural circuit that suppresses appetite. Nature 503: $111-114$

Chua P, Krams M, Toni I, Passingham R, Dolan R (1999). A functional anatomy of anticipatory anxiety. Neuroimage 9: 563-571.

Ciarleglio CM, Resuehr HE, McMahon DG (2011). Interactions of the serotonin and circadian systems: nature and nurture in rhythms and blues. Neuroscience 197: 8-16.

David DJ, Samuels BA, Rainer Q, Wang JW, Marsteller D, Mendez I et al (2009). Neurogenesis-dependent and -independent effects of fluoxetine in an animal model of anxiety/depression. Neuron 62: 479-493.

Farrell MS, Pei Y, Wan Y, Yadav PN, Daigle TL, Urban DJ et al (2013). A Galphas DREADD mouse for selective modulation of cAMP production in striatopallidal neurons. Neuropsychopharmacology 38: 854-862.

Faure C, Mnie-Filali O, Haddjeri N (2006). Long-term adaptive changes induced by serotonergic antidepressant drugs. Expert Rev Neurother 6: 235-245.

Garfield AS, Li C, Madara JC, Shah BP, Webber E, Steger JS et al (2015). A neural basis for melanocortin-4 receptor-regulated appetite. Nat Neurosci 18: 863-871.

Gong S, Doughty M, Harbaugh CR, Cummins A, Hatten ME, Heintz $N$ et al (2007). Targeting Cre recombinase to specific neuron populations with bacterial artificial chromosome constructs. J Neurosci 27: 9817-9823.

Goulding EH, Schenk AK, Juneja P, MacKay AW, Wade JM, Tecott LH (2008). A robust automated system elucidates mouse home cage behavioral structure. Proc Natl Acad Sci USA 105: 20575-20582.

Graeff FG, Guimaraes FS, De Andrade TG, Deakin JF (1996). Role of 5-HT in stress, anxiety, and depression. Pharmacol Biochem Behav 54: 129-141.

Halberstadt AL, Balaban CD (2008). Selective anterograde tracing of nonserotonergic projections from dorsal raphe nucleus to the basal forebrain and extended amygdala. J Chem Neuroanat 35: 317-325.

Jacobs BL, Azmitia EC (1992). Structure and function of the brain serotonin system. Physiol Rev 72: 165-229.

Jain S, Ruiz de Azua I, Lu H, White MF, Guettier JM, Wess J (2013). Chronic activation of a designer $\mathrm{G}(\mathrm{q})$-coupled receptor improves beta cell function. J Clin Invest 123: 1750-1762.

Javanmard M, Shlik J, Kennedy SH, Vaccarino FJ, Houle S, Bradwejn J (1999). Neuroanatomic correlates of CCK-4-induced panic attacks in healthy humans: a comparison of two time points. Biol Psychiatry 45: 872-882.

Kennedy SH, Evans KR, Kruger S, Mayberg HS, Meyer JH, McCann S et al (2001). Changes in regional brain glucose metabolism measured with positron emission tomography after paroxetine treatment of major depression. Am J Psychiatry 158: 899-905.

Krashes MJ, Koda S, Ye C, Rogan SC, Adams AC, Cusher DS et al (2011). Rapid, reversible activation of AgRP neurons drives feeding behavior in mice. J Clin Invest 121: 1424-1428.

Krashes MJ, Shah BP, Madara JC, Olson DP, Strochlic DE, Garfield AS et al (2014). An excitatory paraventricular nucleus to AgRP neuron circuit that drives hunger. Nature 507: 238-242.
Kroenke K, West SL, Swindle R, Gilsenan A, Eckert GJ, Dolor R et al (2001). Similar effectiveness of paroxetine, fluoxetine, and sertraline in primary care: a randomized trial. JAMA 286: 2947-2955.

Lam RW (2012). Onset, time course and trajectories of improvement with antidepressants. Eur Neuropsychopharmacol 22(Suppl 3): S492-S498

Lowry CA, Hale MW, Evans AK, Heerkens J, Staub DR, Gasser PJ et al (2008). Serotonergic systems, anxiety, and affective disorder: focus on the dorsomedial part of the dorsal raphe nucleus. Ann NY Acad Sci 1148: 86-94.

Luyten L, Casteels C, Vansteenwegen D, van Kuyck K, Koole M, Van Laere K et al (2012). Micro-positron emission tomography imaging of rat brain metabolism during expression of contextual conditioning. J Neurosci 32: 254-263.

Marcinkiewcz CA, Dorrier CE, Lopez AJ, Kash TL (2014). Ethanol induced adaptations in 5-HT2c receptor signaling in the bed nucleus of the stria terminalis: implications for anxiety during ethanol withdrawal. Neuropharmacology 89: 157-167.

Mayberg HS, Brannan SK, Tekell JL, Silva JA, Mahurin RK, McGinnis S et al (2000). Regional metabolic effects of fluoxetine in major depression: serial changes and relationship to clinical response. Biol Psychiatry 48: 830-843.

Michaelides M, Anderson SA, Ananth M, Smirnov D, Thanos PK, Neumaier JF et al (2013). Whole-brain circuit dissection in freemoving animals reveals cell-specific mesocorticolimbic networks. I Clin Invest 123: 5342-5350.

Michaelides M, Hurd YL (2015). DREAMM: a biobehavioral imaging methodology for dynamic in vivo whole-brain mapping of cell type-specific functional networks. Neuropsychopharmacology 40: 239-240.

Michaelides M, Pascau J, Gispert JD, Delis F, Grandy DK, Wang GJ et al (2010). Dopamine D4 receptors modulate brain metabolic activity in the prefrontal cortex and cerebellum at rest and in response to methylphenidate. Eur J Neurosci 32: 668-676.

Monti JM (2008). Serotonin and Sleep: Molecular, Functional and Clinical Aspects. Birkhäuser: Basel, Switzerland; Boston MA, USA.

Quitkin FM, Rabkin JG, Ross D, McGrath PJ (1984). Duration of antidepressant drug treatment. What is an adequate trial? Arch Gen Psychiatry 41: 238-245.

Rogan SC, Roth BL (2011). Remote control of neuronal signaling. Pharmacol Rev 63: 291-315.

Santarelli L, Saxe M, Gross C, Surget A, Battaglia F, Dulawa S et al (2003). Requirement of hippocampal neurogenesis for the behavioral effects of antidepressants. Science 301: 805-809.

Sartorius A, Kiening KL, Kirsch P, von Gall CC, Haberkorn U, Unterberg AW et al (2010). Remission of major depression under deep brain stimulation of the lateral habenula in a therapyrefractory patient. Biol Psychiatry 67: e9-e11.

Seminowicz DA, Mayberg HS, McIntosh AR, Goldapple K, Kennedy S, Segal Z et al (2004). Limbic-frontal circuitry in major depression: a path modeling metanalysis. Neuroimage 22: 409-418.

Shumake J, Colorado RA, Barrett DW, Gonzalez-Lima F (2010). Metabolic mapping of the effects of the antidepressant fluoxetine on the brains of congenitally helpless rats. Brain Res 1343: $218-225$

Sohal VS, Zhang F, Yizhar O, Deisseroth K (2009). Parvalbumin neurons and gamma rhythms enhance cortical circuit performance. Nature 459: 698-702.

Stamford JA, Davidson C, McLaughlin DP, Hopwood SE (2000). Control of dorsal raphe 5-HT function by multiple 5-HT(1) autoreceptors: parallel purposes or pointless plurality? Trends Neurosci 23: 459-465.

Straube T, Schmidt S, Weiss T, Mentzel HJ, Miltner WH (2009). Dynamic activation of the anterior cingulate cortex during anticipatory anxiety. Neuroimage 44: 975-981. 
Sung KK, Jang DP, Lee S, Kim M, Lee SY, Kim YB et al (2009). Neural responses in rat brain during acute immobilization stress: a [F-18]FDG micro PET imaging study. Neuroimage 44: 1074-1080.

Underwood MD, Khaibulina AA, Ellis SP, Moran A, Rice PM, Mann JJ et al (1999). Morphometry of the dorsal raphe nucleus serotonergic neurons in suicide victims. Biol Psychiatry 46: 473-483.

Vertes RP (1991). A PHA-L analysis of ascending projections of the dorsal raphe nucleus in the rat. J Comp Neurol 313: 643-668.
Waselus M, Valentino RJ, Van Bockstaele EJ (2012). Collateralized dorsal raphe nucleus projections: a mechanism for the integration of diverse functions during stress. J Chem Neuroanat 41: $266-280$.

Willner P (1990). Animal models of depression: an overview. Pharmacol Ther 45: 425-455.

Wong KP, Sha W, Zhang X, Huang SC (2011). Effects of administration route, dietary condition, and blood glucose level on kinetics and uptake of 18F-FDG in mice. J Nucl Med 52: 800-807.

Supplementary Information accompanies the paper on the Neuropsychopharmacology website (http://www.nature.com/npp) 